Journal of Sports Research

2014. Vol.1, No.2, pp. 34-44

$\operatorname{ISSN}(e): 2410-6534$

$\operatorname{ISSN}(p): 2413-8436$

DOI: 10.18488/journal.90/2014.1.2/90.2.34.44

(C) 2014 Conscientia Beam. All Rights Reserved.

(1) Cossmatk

\title{
THE EFFECTS OF CARBOHYDRATE AND CAFFEINE MOUTH RINSING ON ARM CRANK TIME-TRIAL PERFORMANCE
}

\author{
Jonathan Sinclair ${ }^{1 *}$--- Lindsay Bottoms ${ }^{2}$ \\ Centre for Applied Sport and Exercise Sciences, University of Central Lancashire, United Kingdom \\ ${ }^{2}$ School of Health, Sport and Bioscience, University of East London
}

\begin{abstract}
This study aimed to determine whether carbohydrate (CHO) and caffeine (CAFF) mouth rinsing would improve 30 minute arm cranking time-trial performance. Twelve male participants (age $21.54 \pm 1.28$ years, height $179.46 \pm 7.38 \mathrm{~cm}$ and mass $73.69 \pm 5.40 \mathrm{~kg})$ took part in the current investigation. Participants came to the laboratory on 3 occasions during which they performed 30 minute self-paced arm crank time trials. On one occasion water was given as a mouth rinse for $5 s$ (PLA), on another occasion a $6.4 \% \mathrm{CHO}$ solution was given for $5 \mathrm{~s}$ and finally a $0.032 \% \mathrm{CAFF}$ solution was given for 5 s. Key measurements of distance covered, heart rate (HR), ratings of perceived exertion (RPE), cadence and power output were recorded throughout all trials. Distance covered during the CAFF (15.43 $\pm 3.27 \mathrm{~km})$ and CHO (15.30 \pm 3.31$)$ mouth rinse trials were significantly $(p<0.05)$ greater in comparison to PLA $(13.15$ $\pm 3.36 \mathrm{~km})$. Cadence and power output and velocity were also significantly greater during the CAFF and CHO trials compared to PLA and CHO $(p<0.05)$. No significant $(P>0.05)$ differences between trials were observed for HR and RPE. CAFF and CHO mouth rinse serve to improve 30 minute arm cranking performance by mediating increasing cadence and power output without a concurrent increase in RPE and HR.
\end{abstract}

Keywords: Mouth rinse, Carbohydrate, Caffeine, Arm cranking, Physiology.

Received: 10 December 2014/ Revised: 23 December 2014/ Accepted: 30 December 2014/ Published: 2 January 2015

\section{Contribution/ Originality}

This study contributes in the existing literature as the first investigation to comparatively examine the influence of both carbohydrate and caffeine mouth rinsing on upper body time trial performance.

\section{INTRODUCTION}

There is now a considerable body of evidence indicating that the ingestion of both carbohydrate $(\mathrm{CHO})$ and caffeine $(\mathrm{CAFF})$ serve to improve endurance exercise performance 
(Costill et al., 1978; Angus et al., 2000; Cox et al., 2002; Andrews et al., 2003; Jacobs et al., 2003; Desbrow et al., 2004; Doherty and Smith, 2005; Beck et al., 2006; Astorino et al., 2008; Beaven et al., 2008; Burke, 2008; Osterberg et al., 2008; Hulston and Jeukendrup, 2009; Ganio et al., 2010). Endurance exercise has been shown to reduce the body's glycogen stores (Greiwe et al., 1999), as such supplementing with $\mathrm{CHO}$ may be a mechanism by which the onset of fatigue can be delayed (Coyle et al., 1983). Glycogen depletion as a function of exercise is the key mechanism by which sports performance decrements are mediated (Williams et al., 2012). Therefore, increased CHO ingestion availability has been advocated as a method of maintaining euglycaemia and blood oxidation rates (Tsintzas and Williams, 1998), thus allowing higher exercise intensities to be maintained during exercise. The ergogenic benefits of CAFF are attributed to its affinity with adenosine receptors. CAFF is able to bind to both pre and post synaptic receptors, which serve to inhibit adenosine action (Davis and Green, 2009). This promotes the discharge of excitatory neurotransmitters which enhance corticomotor sensitivity (Cerqueira et al., 2006). The stimulation of central effects by CAFF may be responsible for modifying the motivational factors that affect discomfort during exercise, leading to a reduction in perceived exertion/ pain during exercise (Kalmar and Cafarelli, 2004). This mechanism may also facilitate the release of calcium $\left(\mathrm{Ca}^{2+}\right)$ ions from the sarcoplasmic reticulum, which allows more frequent and forceful muscular contractions to take place (Klein et al., 1990).

In addition to the aforementioned mechanisms, there is now evidence to suggest that the ingestion of ergogenic aids may not be necessary in order to mediate improvements in athletic performance. Rinsing $\mathrm{CHO}$ in the mouth has been shown to improve high intensity exercise performance (Chambers et al., 2009; Pottier et al., 2010; Rollo et al., 2010; Sinclair et al., 2014). The mechanism by which $\mathrm{CHO}$ rinsing is thought to improve exercise is through carbohydrate mouth receptors which regulate the central mechanisms linked to motivation (Chambers et al., 2009). Furthermore, recent research has established that CAFF can be absorbed in the buccal mucosa (Kamimori et al., 2012) leading to research being undertaken in to CAFF chewing gum on cycling performance (Paton et al., 2010; Ryan et al., 2013). This suggests that caffeine mouth rinse will also improve exercise performance. This was confirmed by Beaven et al. (2013) who showed that rinsing a $1.2 \% \mathrm{CAFF}$ mouth rinse solution improved repeated sprint cycling performance. In addition Bottoms et al. (2014) demonstrated that rinsing a $0.032 \%$ CAFF solution served to significantly enhance 30 min cycling time trial performance.

However, despite the sizeable body of evidence that now exists regarding the ergogenic benefits of both $\mathrm{CHO}$ and $\mathrm{CAFF}$ rinsing; the majority of these investigations have considered only lower body exercise. Therefore, the effects of both $\mathrm{CHO}$ and $\mathrm{CAFF}$ mouth rinsing on upper body exercise are not yet known. Typically the upper body has a greater proportion of type II muscles fibres in comparison to the lower extremities (Bernasconi et al., 2006). Type I fibres which are predominant in the lower body are metabolically more efficient than Type II fibres (Smith et al., 2006). Upper body exercise therefore necessitates a larger $\mathrm{O}_{2}$ cost than does lower body exercise to produce the power output (Smith et al., 2006). As there are known differences in 
muscle fibre contributions between upper and lower body exercise $\mathrm{CHO}$ and $\mathrm{CAFF}$ rinsing may have differential effects on different exercise modalities (Bottoms et al., 2014). Thus the observations from previous investigations which have focused on the lower body cannot be generalized to upper body exercise.

The aim of the current study was therefore to determine whether $\mathrm{CHO}$ and CAFF have any effect on 30 min arm crank time trial performance. A study of this nature will provide further insight into the ergogenic benefits of both $\mathrm{CHO}$ and $\mathrm{CAFF}$ rinsing. The current investigation tests the hypothesis that both $\mathrm{CHO}$ and $\mathrm{CAFF}$ rinsing will serve to significantly enhance upper body exercise in comparison to placebo (PLA).

\section{METHODS}

\subsection{Participants}

Twelve male participants (age 21.54 \pm 1.28 years, height $179.46 \pm 7.38 \mathrm{~cm}$ and mass $73.69 \pm$ $5.40 \mathrm{~kg}$ ) took part in this investigation. Participants were free from musculoskeletal injury and provided written informed consent. The investigation was approved by the University of Central Lancashire, School of Sport Tourism and Outdoors, ethical committee in accordance with the principles detailed in the declaration of Helsinki.

\subsection{Procedure}

Data collection was accomplished using an arm crank ergometer (MONARK, Cardio Rehab $891 \mathrm{E}$, Nottingham). The data collection procedure required a total of four visits to a bespoke exercise physiology laboratory. Visit 1 served as a familiarization session, whilst visits $2-4$ were used for data collection in which participants performed 30 min time trials using the arm crank ergometer for maximum distance. For data collection i.e. sessions 2-4 all participants were administered either $4 \times 25 \mathrm{ml}$ of a tasteless $6.4 \%$ maltodextrin (Maltodextrin, My Protein) solution (CHO), $0.032 \%$ caffeine (MyProtein; this was representative of the caffeine concentrations observed in commercially available caffeinated drinks and in accordance with Bottoms et al. (2014) solution (CAFF) or a water bolus (PLA).

\section{Visit 1}

The first visit to the laboratory represented a familiarisation session during which a $30 \mathrm{~min}$ time trial was performed using the same protocol as in the experimental trials.

\section{Visit 2-4}

Participants were instructed to report to the laboratory having refrained from alcohol, caffeine and exercise in the 24 hours prior and also having fasted for 4 hours ${ }^{35}$. Experimental data was obtained at the same time of day to avoid physiological fluctuations caused by circadian rhythmicity. Participants were firstly fitted with a heart rate monitor (Polar RS100, Polar Electro

Oy Finland). They were asked to arm crank as far as possible in $30 \mathrm{~min}$ at a resistance of $1 \mathrm{~kg}$ 
added to the arm crank ergometer. The ergometer was interfaced with a computer which calculated the outcome measures of heart rate (HR), cadence (strokes.min ${ }^{-1}$ ), power output (W) and distance covered $(\mathrm{m})$ which were quantified at 6-min intervals throughout the 30-min time trials. In addition, participants were also asked to rate their perceived exertion (RPE) using the 6to 20-point Borg scale (Borg, 1982)

at 6-min intervals.

\subsection{Mouth Rinse Administration}

Each participant was given a $25 \mathrm{ml}$ bolus of a tasteless CHO, CAFF or PLA every 6 minutes. The solutions were rinsed for a total of $5 \mathrm{~s}$ and then spat into a bowl. The mouth rinses were controlled using a single-blind mechanism and the order in which they were administered was counterbalanced.

\subsection{Blinding Efficacy}

For the CHO trials 5 of the 12 participants correctly identified that they were receiving an ergogenic solution and for the CAFF trials 6 of the 12 participants were able to identify that they were receiving an ergogenic solution.

\subsection{Statistical Analysis}

To examine any effects of CHO, CAFF and PLA mouth rinsing 5 x 3 (time $\mathrm{x}$ condition) repeated measures ANOVA's were conducted. Main effects were further explored using post-hoc pairwise comparisons and significant interactions were examined using simple main effects. Statistical significance throughout was accepted at the $\mathrm{p}<0.05$ level. Effect sizes were calculated using partial $\mathrm{Eta}^{2}\left(\mathrm{p \eta}^{2}\right)$. All statistical procedures were conducted using SPSS v22.0 (SPSS Inc., Chicago, IL, USA).

\section{RESULTS}

\subsection{Distance Covered}

Of the twelve participants 10 and 9 were able to complete a greater distance in the $\mathrm{CHO}$ and CAFF conditions respectively in comparison to PLA. Significant main effects were observed for both condition $\left(\mathrm{p}<0.05, \mathrm{p} \eta^{2}=0.30\right)$ and time $\left(\mathrm{p}<0.05, \mathrm{p} \eta^{2}=0.96\right)$. In addition a significant interaction $\left(\mathrm{p}<0.05, \mathrm{p} \eta^{2}=0.27\right)$ was also observed between condition and time. Further analysis using simple main effects showed significant differences between conditions at $6 \min \left(\mathrm{p}<0.05, \mathrm{p} \eta^{2}\right.$ $=0.26) 12 \min \left(\mathrm{p}<0.05, \mathrm{p} \eta^{2}=0.28\right), 18 \min \left(\mathrm{p}<0.05, \mathrm{p}^{2}=0.26\right), 24 \min \left(\mathrm{p}<0.05, \mathrm{p} \eta^{2}=0.25\right)$ and $30 \min \left(\mathrm{p}<0.05, \mathrm{p} \eta^{2}=0.30\right)$ time points. Specifically the results indicated that distances covered during the $\mathrm{CHO}$ and $\mathrm{CAFF}$ trials were significantly greater than PLA at each of the time points. Overall distance covered was shown to be $15.30 \pm 3.31 \mathrm{~km}$ in the $\mathrm{CHO}, 15.43 \pm 3.27 \mathrm{~km}$ in the $\mathrm{CAFF}$ and $13.15 \pm 3.36 \mathrm{~km}$ in the PLA trials (Figure 1). 


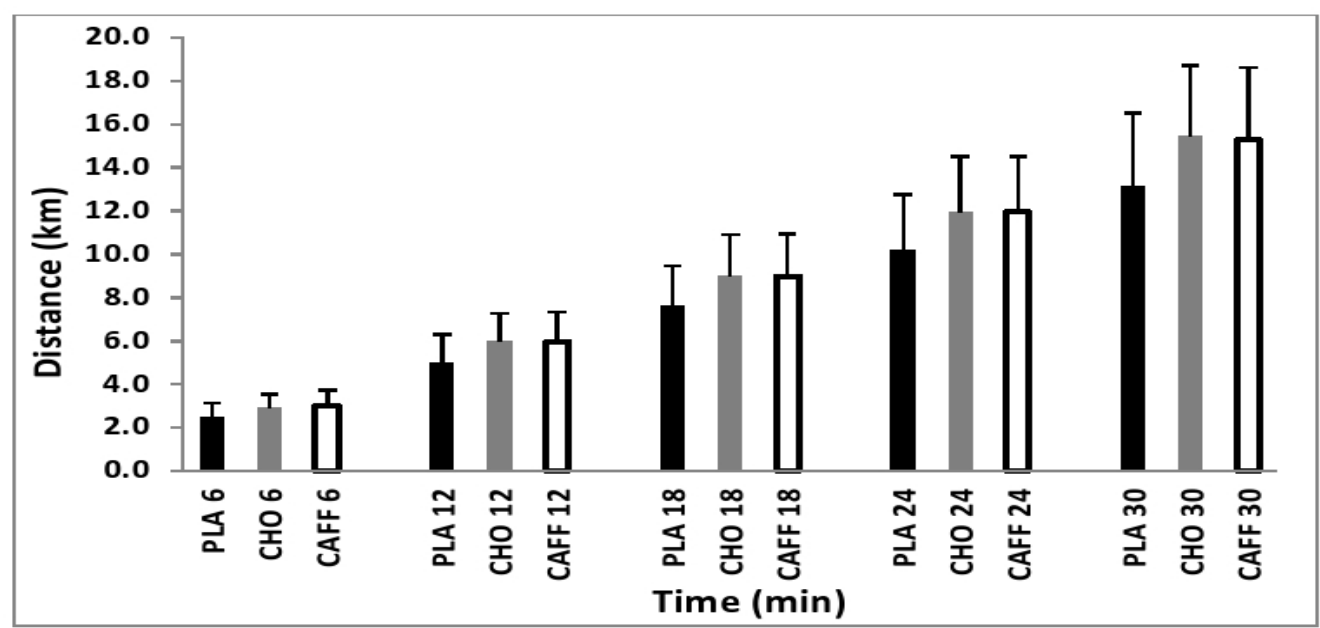

Figure-1. Mean $( \pm \mathrm{SD})$ distance completed in 30 minutes during each condition.

\subsection{Cadence and Power Output}

For power output there was a significant main effect for time $\left(\mathrm{p}<0.05, \mathrm{p} \eta^{2}=0.52\right)$. Post-hoc analysis revealed that power output at 30 min was significantly $(\mathrm{p}<0.05)$ greater than any of the other time points. There was also a main effect for condition $\left(\mathrm{p}<0.05, \mathrm{p} \eta^{2}=0.34\right)$ for which post hoc analysis showed that power output was significantly greater $(\mathrm{p}<0.05)$ in the CAFF and CHO trials compared to PLA (Figure 2a). For cadence there was also a significant main effect for time $\left(\mathrm{p}<0.05, \mathrm{p}^{2}=0.55\right)$. Post-hoc analysis revealed that power output at $30 \mathrm{~min}$ was significantly $(\mathrm{p}<0.05)$ greater than any of the other time points. There was also a main effect for condition $\left(\mathrm{p}<0.05, \mathrm{p}^{2}=0.33\right)$ for which post hoc analysis showed that cadence was significantly larger $(\mathrm{p}<0.05)$ in the CAFF and CHO trials compared to PLA (Figure $2 \mathrm{~b})$.

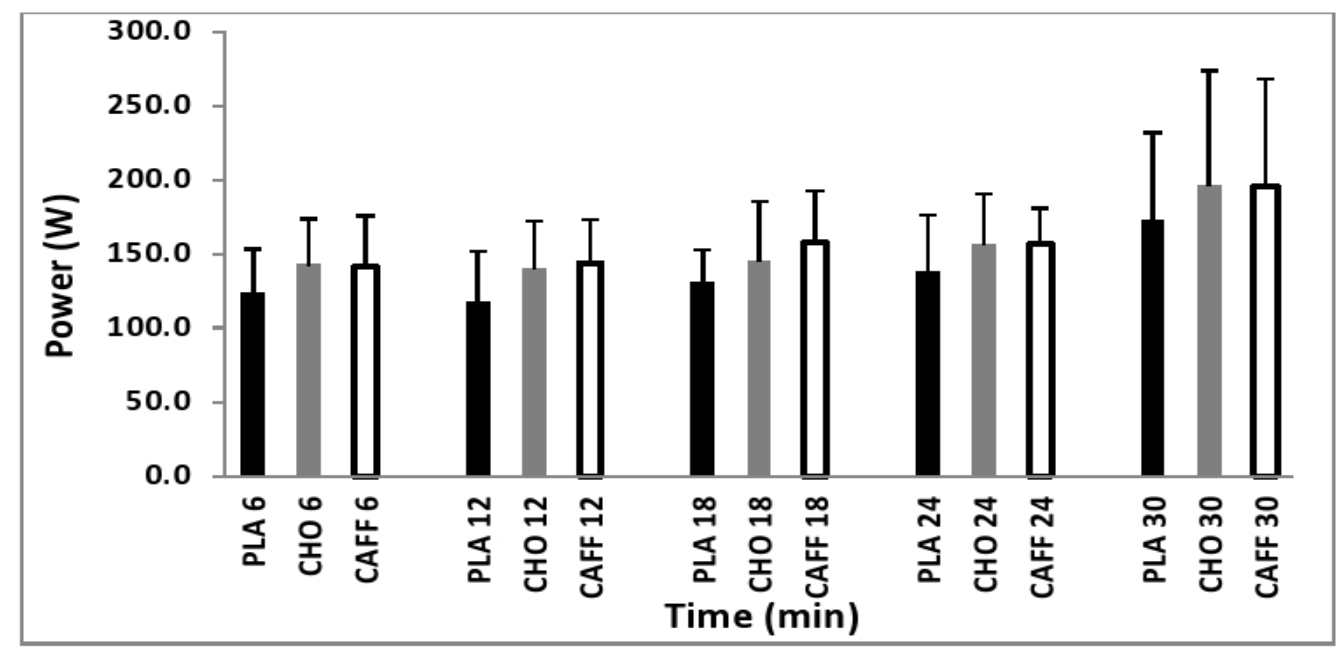

Figure-2a. Mean $( \pm \mathrm{SD})$ power output during the 30 minute exercise for each condition. 


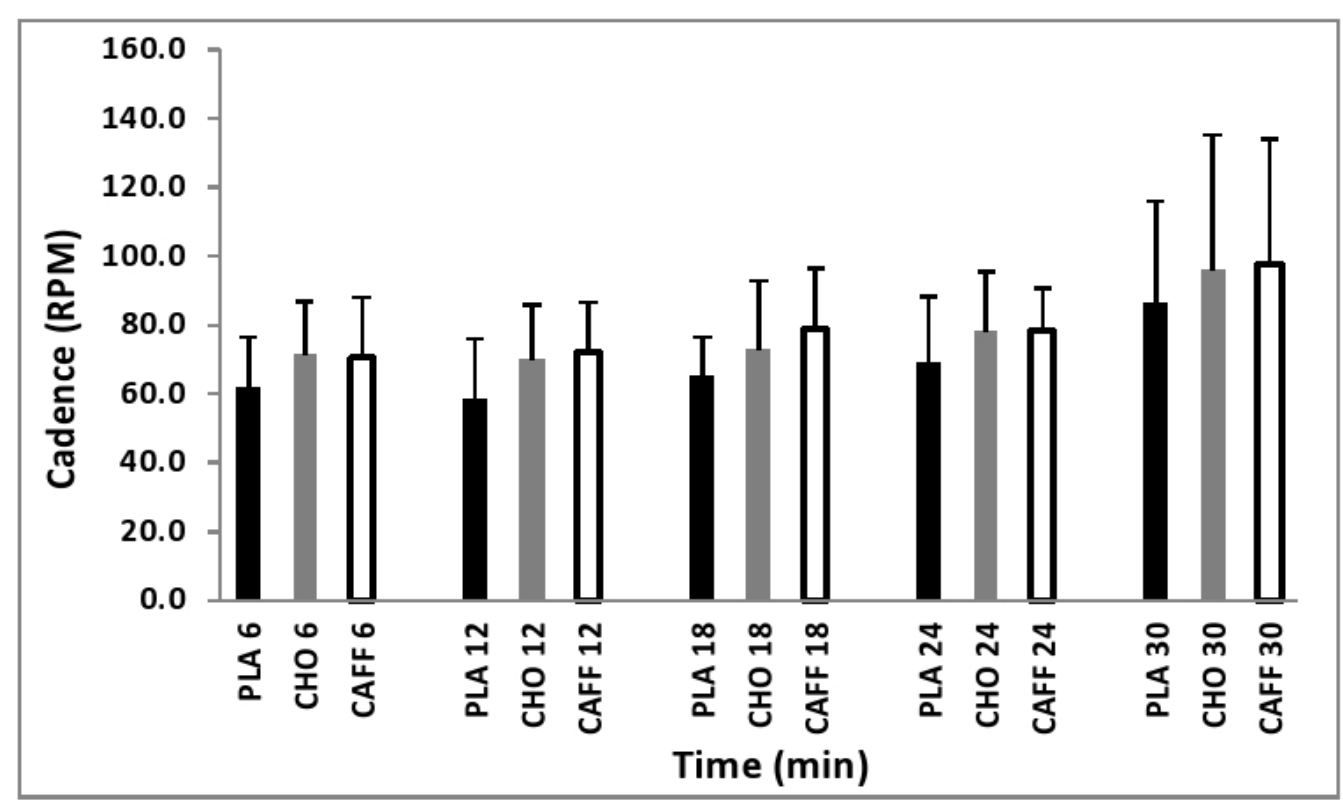

Figure-2b. Mean $( \pm \mathrm{SD})$ cadence during the 30 minute exercise for each condition.

\subsection{Heart Rate and RPE}

For HR there was a significant main effect for time $\left(\mathrm{p}<0.05, \mathrm{p} \eta^{2}=0.60\right)$. Post-hoc analysis revealed that HR at 24 and 30 min were significantly $(\mathrm{p}<0.05)$ greater than any of the other time points (Figure 3a). For RPE there was also a significant main effect for time $\left(\mathrm{p}<0.05, \mathrm{p} \eta^{2}=0.76\right)$. Post-hoc analysis revealed that RPE increased linearly as a function of time and that each time point different significantly $(\mathrm{p}<0.05)$ from the others (Figure $3 \mathrm{~b})$.

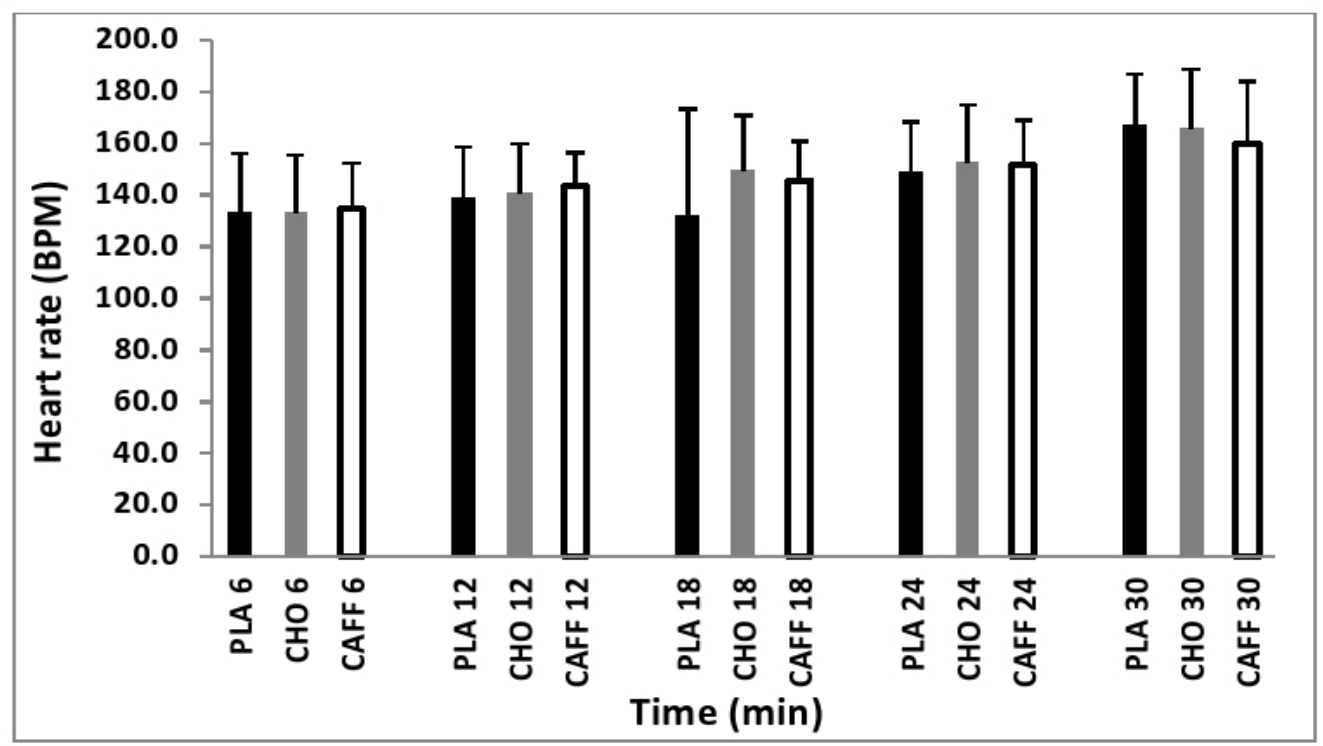

Figure-3a. Mean $( \pm \mathrm{SD})$ heart rate during the 30 minute exercise for each condition. 


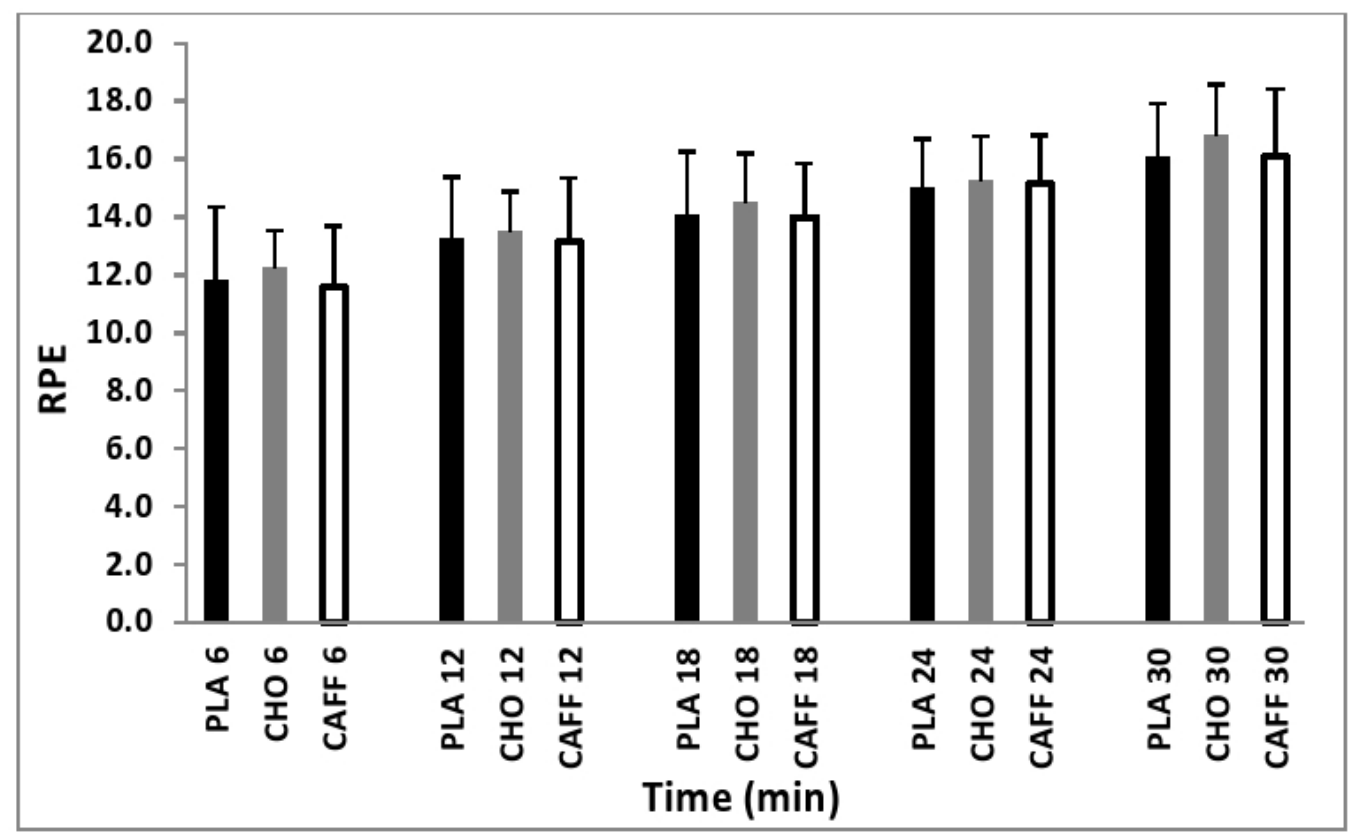

Figure-3b. Mean $( \pm \mathrm{SD})$ RPE during the 30 minute exercise for each condition.

\section{DISCUSSION}

The aim of the current investigation was to explore the effects of $\mathrm{CHO}$ and $\mathrm{CAFF}$ mouth rinsing on upper body exercise. This represents the first investigation to comparatively examine the influence of both $\mathrm{CHO}$ and $\mathrm{CAFF}$ mouth rinsing on upper body time trial performance.

In support of our hypothesis the results demonstrate that both $\mathrm{CAFF}$ and $\mathrm{CHO}$ mouth rinsing increased distance covered during the 30 minutes of self-paced arm cranking. This observation supports those that have been observed previously in lower body exercise regarding the positive performance influence of $\mathrm{CHO}$ mouth rinsing on high intensity exercise performance (Chambers et al., 2009; Pottier et al., 2010; Rollo et al., 2010; Sinclair et al., 2014). The results from this study also support those of Beaven et al. (2013) and Bottoms et al. (2014) who found that $\mathrm{CAFF}$ mouth rinsing improved performance in high intensity cycling. The key implication from the current investigation is that $\mathrm{CHO}$ and $\mathrm{CAFF}$ rinsing appear to also be beneficial to those undertaking upper body exercise.

There was a $17 \%$ improvement in performance during the $\mathrm{CHO}$ trial, which is by far greater than for lower body exercise trials which have previously found increases of around 2-3\% (Chambers et al., 2009; Pottier et al., 2010; Rollo et al., 2010; Sinclair et al., 2014). This greater increase in performance could be due to the differences in musculature (the upper body has a greater percentage of Type II fibres compared to the lower body) as well as the fact submaximal arm exercise is metabolically less efficient and produces greater respiratory variables and perceived exertion (Ahlborg et al., 1986). These differences in physiological responses between the upper and lower body musculature suggest that carbohydrate utilisation is greater during work 
with the arms than the legs. Therefore, when carbohydrate activates the anterior cingulated cortex and ventral striatum as well as other brain regions during mouth rinsing which has previously been observed by Chambers et al. (2009) it could potentially stimulate a stronger increase in performance due to the increased demand for carbohydrate as fuel. In comparison, there was a $16 \%$ improvement in performance during the CAFF trial, which is similar to those found in lower body performance by meta-analyses by Doherty and Smith (2005) and Ganio et al. (2010) who found increases in endurance capacity of $12.3 \%$. Caffeine has been observed to be absorbed rapidly in the buccal mucosa (Kamimori et al., 2012) which has led to researchers using caffeine chewing gum to observe improvements in cycling performance (Paton et al., 2010; Ryan et al., 2013). Caffeine could potentially be improving performance by decreasing perceived exertion and reducing pain perception due to the action of adenosine and dopamine acting on the brain (Meeusen et al., 2013).

The current study confirmed that no differences in RPE were observed between rinsing conditions despite the significant increase in distance covered in the CAFF and CHO trials. This finding is important as it shows that participants were able to exercise at a higher intensity but at a similar RPE in the CAFF and CHO conditions. This supports the findings of Pottier et al. (2010), who showed that participants were able to produce a larger external power output for the same level of perceived discomfort. This indicates that there may have been an increase in motivation during the $\mathrm{CHO}$ and CAFF trials (Kalmar and Cafarelli, 2004). The physiological mechanisms responsible for the positive effects of both $\mathrm{CHO}$ and $\mathrm{CAFF}$ on exercise performance are not yet fully understood. Chambers et al. (2009) utilized functional magnetic resonance techniques to study brain activation during carbohydrate mouth rinsing and determined that a $\mathrm{CHO}$ mouth rinse enhanced activation of the areas in the brain linked to motivation. The findings from the current investigation regarding performance benefits of CAFF rinsing indicate that further investigation is warranted using magnetic resonance imaging to determine whether similar brain functions are mediated when using a $\mathrm{CAFF}$ compared to $\mathrm{CHO}$ rinse or whether improvements are a result of absorption in the buccal mucosa.

The results from the current study may have important practical benefits for those who habitually partake in high intensity upper body exercise. It appears based on these observations that when undertaking upper body exercise athletes may benefit from rinsing instead of ingesting both $\mathrm{CHO}$ and $\mathrm{CAFF}$ solutions. Ingestion of both $\mathrm{CHO}$ and $\mathrm{CAFF}$ has been linked to gastrointestinal issues during exercise (Peters et al., 1993; Boekema et al., 1999). Therefore rinsing $\mathrm{CAFF}$ and $\mathrm{CHO}$ may allow athletes to have benefit from these ergogenic solutions but also avoid the gastrointestinal distress associated with their ingestion. Finally mouth rinsing $\mathrm{CHO}$ and CAFF could also be a mechanism by which athletes who have diabetes or are sensitive to caffeine could benefit from the ergogenic effects of $\mathrm{CHO}$ and $\mathrm{CAFF}$ without the detrimental health consequences (Sinclair et al., 2014).

In conclusion, although previous analyses have investigated the effects of both CAFF and $\mathrm{CHO}$ rinsing these studies had all been confined to lower body exercise modalities. The current 
investigation addresses this gap in the literature by providing a comprehensive investigation of the effects of both $\mathrm{CAFF}$ and $\mathrm{CHO}$ mouth rinse on upper body exercise performance. This study provides evidence to suggest that both $\mathrm{CHO}$ and $\mathrm{CAFF}$ rinse can improve $30 \mathrm{~min}$ arm cranking performance. The underlying mechanisms behind these improvements in performance in the absence of ingestion are still not fully understood at the current time and future work should seek determine the physiological mechanisms by which mouth rinsing exerts its effects. Nonetheless, this work confirms that those performing in short duration upper body exercise based events may be able to improve their resultant performance through utilization of a $\mathrm{CHO}$ of $\mathrm{CAFF}$ mouth rinse.

Funding: This study received no specific financial support.

Competing Interests: The authors declare that they have no competing interests.

Contributors/Acknowledgement: All authors contributed equally to the conception and design of the study.

\section{REFERENCES}

Ahlborg, G., J. Wahren and P. Felig, 1986. Splanchnic and peripheral glucose and lactate metabolism during and after prolonged arm exercise. J Clin Invest, 77 (3): 690-699.

Andrews, J., D. Sedlock, M. Flynn, J. Navalta and H. Ji, 2003. Carbohydrate loading and supplementation in endurance-trained women runners. J Appl Physiol, 95 (52): 584-590.

Angus, D., M. Hargreaves, J. Dancey and M. Febbraio, 2000. Effect of carbohydrate or carbohydrate plus medium-chain triglyceride ingestion on cycling time trial performance. J Appl Physiol, 88 (1): 113119 .

Astorino, T., K. Firth and R. Rohmann, 2008. Effect of caffeine ingestion on one-repetition maximum muscular strength. Eur J Appl Physiol., 102 (2): 127-132.

Beaven, C., W. Hopkins, K. Hansen, M. Wood, J. Cronin and T. Lowe, 2008. Dose effect of caffeine on testosterone and cortisol responses to resistance exercise. Int J Sport Nutr Exerc Metab., 18 (2): 131-141.

Beaven, C., P. Maulder, A. Pooley, L. Kilduff and C. Cook, 2013. Effects of caffeine and carbohydrate mouth rinses on repeated sprint performance. Appl Physiol Nutr Metab., 38 (6): 633-637.

Beck, T., T. Housh, R. Schmidt, G. Johnson, D. Housh, J. Coburn and M. Malek, 2006. The acute effects of a caffeine containing supplement on strength, muscular endurance, and anaerobic capabilities. J Strength Cond Res., 20 (3): 506-510.

Bernasconi, S., N. Tordi, S. Perrey, B. Parratte and G. Monnier, 2006. Is the VO2 slow component in heavy arm-cranking exercise associated with recruitment of type II muscle fibers as assessed by an increase in surface EMG? Appl Physiol Nutr Metab., 31 (4): 414-422.

Boekema, J., G. Samsom, A. Van Berge Henegouwen and P. Smout, 1999. Coffee and gastrointestinal function: Facts and fiction: A review. Scand Journal Gast., 34: 35-39.

Borg, G., 1982. Psychophysical bases of perceived exertion. Med Sci Sports Exerc., 14 (5): 377-381. 
Bottoms, L., H. Hurst, A. Scriven, F. Lynch, J. Bolton, L. Vercoe, Z. Shone, G. Barry and J. Sinclair, 2014. The effect of caffeine mouth rinse on self-paced cycling performance. Comp Ex Phys., 10 (4): 239245.

Burke, L., 2008. Caffeine and sports performance. Appl Physiol Nutr Metab., 33 (6): 1319-1334.

Cerqueira, V., A. De Mendonça, A. Minez, A. Dias and M. De Carvalho, 2006. Does caffeine modify corticomotor excitability? Neurophysiol Clin., 36 (4): 219-226.

Chambers, E., M. Bridge and D. Jones, 2009. Carbohydrate sensing in the human mouth: Effects on exercise performance and brain activity. J Physiol., 587: 1779-1794.

Costill, D., G. Dalsky and W. Fink, 1978. Effects of caffeine on metabolism and exercise performance. Med Sci Sports Exerc., $10(3):$ 155-158.

Cox, G., B. Desbrow, P. Montgomery, M. Anderson, C. Bruce, T. Macrides, D. Martin, A. Moquin, A. Roberts, J. Hawley and L. Burke, 2002. Effect of different protocols of caffeine intake on metabolism and endurance performance. J Appl Physiol., 93 (3): 990-999.

Coyle, E., J. Hagberg, B. Hurley, W. Martin, A. Ehsani and J. Holloszy, 1983. Carbohydrate feeding during prolonged strenuous exercise can delay fatigue. J App Phys: Resp, Env and Exerc Phys., 55 (1): 230-235.

Davis, J. and J. Green, 2009. Caffeine and anaerobic performance ergogenic value and mechanisms of action. Sports Med., 39 (10): 813-832.

Desbrow, B., S. Anderson, J. Barrett, E. Rao and M. Hargreaves, 2004. Carbohydrate-electrolyte feedings and $1 \mathrm{~h}$ time trial cycling performance. Int J Sport Nutr Exerc Metab., 14 (5): 541-549.

Doherty, M. and P. Smith, 2005. Effects of caffeine ingestion on rating of perceived exertion during and after exercise: A meta-analysis. Scand J Med Sci Sports, 15 (2): 69-78.

Ganio, M., J. Klau, E. Lee, S. Yeargin, B. McDermott, M. Buyckx, C. Maresh and L. Armstrong, 2010. Effect of various carbohydrate-electrolyte fluids on cycling performance and maximal voluntary contraction. Int J Sport Nutr Exerc Metab., 20 (2): 104-114.

Greiwe, J., R. Hickner, P. Hansen, S. Racette, M. Chen and J. Holloszy, 1999. Effects of endurance exercise training on muscle glycogen accumulation in humans. Journal App Phys., 87 (1): 222-226.

Hulston, C. and A. Jeukendrup, 2009. No placebo effect from carbohydrate intake during prolonged exercise. Int J Sport Nutr Exerc Metab., 19 (3): 275-284.

Jacobs, I., H. Pasternak and D. Bell, 2003. Effects of ephedrine, caffeine, and their combination of muscular endurance. Med Sci Sports Exerc., 35 (6): 987-994.

Kalmar, J. and E. Cafarelli, 2004. Caffeine: A valuable tool to study central fatigue in humans? Exerc Sport Sci Rev., $32(4): 143-147$.

Kamimori, G., C. Karyekar, R. Otterstetter, D. Cox, T. Balkin, G. Belenky and N. Eddington, 2012. The rate of absorption and relative bioavailability of caffeine administered in chewing gum versus capsules to normal healthy volunteers. Int Jou of Pharm., 234 (1): 159-167.

Klein, M., B. Simon and M. Schneider, 1990. Effects of caffeine on calcium release from the sarcoplasmic reticulum in frog skeletal muscle fibres. Journal Phys., 425: 599-626.

Meeusen, R., B. Roelands and L. Spriet, 2013. Caffeine, exercise and the brain. Nestle Nutrition Institute Workshop Series, 76: 1-12. 
Osterberg, K., J. Zachwieja and J. Smith, 2008. Carbohydrate and carbohydrate + protein for cycling timetrial performance. J Sports Sci., 26 (3): 227-233.

Paton, C., T. Lowe and A. Irvine, 2010. Caffeinated chewing gum increases repeated sprint performance and augments increases in testosterone in competitive cyclists. Eur J App Phys., 110 (6): 1243-1250.

Peters, H., F. van Schelven, P. Verstappen, R. De Boer, E. Bol, W. Erich and W. De Vries, 1993. Gastrointestinal problems as a function of carbohydrate supplements and mode of exercise. Med Sci Sports Exerc., 25 (11): 1211-1224.

Pottier, A., J. Bouckaert, W. Gilis, T. Roels and W. Derave, 2010. Mouth rinse but not ingestion of a carbohydrate solution improves 1-h cycle time trial performance. Scand J Med Sci Sports, 20 (1): 105-111.

Rollo, I., M. Cole, R. Miller and C. Williams, 2010. Influence of mouth rinsing a carbohydrate solution on 1h running performance. Med Sci Sports Exer., 42 (4): 798-804.

Ryan, E., C. Kim, E. Fickes, M. Williamson, M. Muler, J. Barkley and E. Glickman, 2013. Caffeine gum and cycling performance: A timing study. J Strength Cond Res., 27 (1): 259-264.

Sinclair, J., L. Bottoms, C. Flynn, E. Bradley, G. Alexander, S. McCullagh and H. Hurst, 2014. The effect of different durations of carbohydrate mouth rinse on cycling performance. Eur J Sport Sci., 14 (3): 259-264.

Smith, P., E. McCrindle, M. Doherty, M. Price and A. Jones, 2006. Influence of crank rate on the slow component of pulmonary $\mathrm{O} 2$ uptake during heavy arm-crank exercise. Appl Physiol Nutr Metab., 31 (3): 292-301.

Tsintzas, K. and C. Williams, 1998. Human muscle glycogen metabolism during exercise; effect of carbohydrate supplementation. Sports Med., 25 (1): 7-23.

Williams, J., T. Batts and S. Lees, 2012. Reduced muscle glycogen differentially affects exercise performance and muscle fatigue. Int Schol Res Not, 13: 1-8.

Views and opinions expressed in this article are the views and opinions of the author(s), Journal of Sports Research shall not be responsible or answerable for any loss, damage or liability etc. caused in relation to/arising out of the use of the content. 